\title{
Research Paper: The Effect of Educational Intervention Based on Orem Self-Care Model on Family Caregiver's Burden of Patients with Spinal Cord Injuries
}

\author{
Somayeh Mohammadi ${ }^{1}$, ${ }^{*}$ Mostafa Roshanzadeh ${ }^{2}$ \\ 1. Ayatollah Kashani Hospital, Shahrekord University of Medical Sciences, Shahrekord, Iran. \\ 2. Surgical Care Research Center, Birjand University of Medical Sciences, Birjand, Iran.
}

citeat on: Mohammadi S, Roshanzadeh M. [The effect of educational intervention based on Orem self-care model on family caregiver's burden of patients with spinal Cord Injuries (Persian)]. Journal of Rehabilitation. 2016; 17(1):54-63. http://dx.doi.org/10.20286/jrehab-170152

do: $:$ http://dx.doi.org/10.20286/jrehab-170152

Received: 4 Jul. 2015 Accepted: 7 Oct. 2015
Keywords:

Self-care, Burden, Family caregivers, Spinal cord injuries

\section{ABSTRACT}

Objective Family member caregivers of patients with spinal cord injuries experience varying degrees of burden because of the long process in the care of their patients. These conditions have different negative consequences for the family caregivers. One of the most important protective factors against the suffering of the caregivers is their knowledge about care giving process. Therefore, the aim of the present study was to evaluate the effect of educational intervention based on Orem self-care model on family caregivers' burden of patients with spinal cord injuries.

Materials \& Methods This was a quasi-experimental study with pretest and posttest design and control group. It was conducted on patients with spinal cord injuries at South Khorasan Province. Research units purposefully selected and then were randomly allocated to experimental and control groups. Experimental group received educational intervention based on Orem self-care model for patients with spinal cord injuries consisted of eight 40-minute teaching sessions at the center. To measure the burden of caregivers, 22-item Zarit questionnaire was used with content validity index of $80 \%$ and Cronbach $\alpha$ of 0.79. The questionnaire was completed before and after the intervention in experimental and control groups. The results were analyzed by SPSS version 16 using descriptive (frequency, mean and standard deviation) and analytical (t-test, ANOVA and Pearson correlation coefficient) statistics.

Results Mean of burden in caregivers was not significantly different between the 2 groups before The intervention. But, we observed significant differences between the 2 groups after the intervention. The difference between mean of burden before $(3.2 \pm 0.33)$ and after $(3.11 \pm 0.66)$ the intervention was significant $(P<0.05)$ which shows the positive impact of education. The results obtained with regard to the relationship between mean burden of caregivers and demographic variables indicate that there is a significant relationship between the burden of caregivers and their income, before and after intervention in 2 groups $(P<0.05)$. Furthermore, burden score shows a significant positive association with the duration of caring the damaged patient. The longer the duration of caring, the higher would be the burden score $(P<0.05, r=0.3)$. The burden score relates also with increasing age, both positively and significantly $(P<0.05, r=0.3)$. There was no significant correlation between other variables and burden score $(P>0.05)$. Conclusion The results of this study suggest that education of family caregivers of patients with spinal cord injuries based on Orem self-care model can reduce their experienced burden resulted from chronic and long term caregiving involvement. Moreover, the financial situation and occupation of caregivers could be a positive relational factor in their burden. Therefore, we suggest that the family of caregivers be under support and supervision of social and therapeutic supportive organizations. Also, with regard to long-term care of these patients, some measures like caring the patient at home with support and backing of spinal-cord injury centers to reduce the pressure on the family of caregivers are recommended.

\section{* Corresponding Author:}

Mostafa Roshanzadeh, PhD

Address: Surgical Care Research Center, Birjand University of Medical Sciences, Birjand, South Khorasan, Iran.

Tel: +98 (939) 7952522

E-Mail: mroshanzadeh62@gmail.com 


\title{
تأثير آموزش مطابق التَوى خودمراقبتى اورِم بر سطح مشقت و رنج عضو خانواده مراقبت كننده از

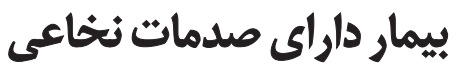

\author{
سميه محمدى'!، "مصطفى روشنزاده

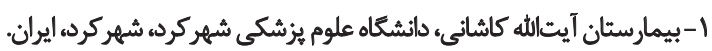

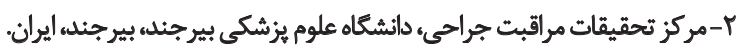

\begin{abstract}
تاريخ دريافت: تانتير

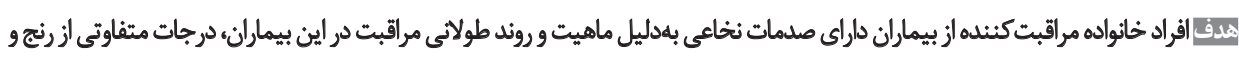

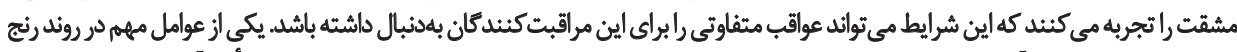

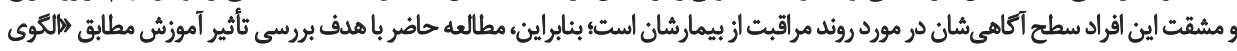

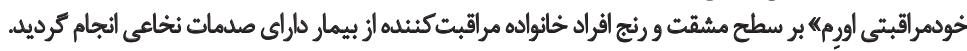

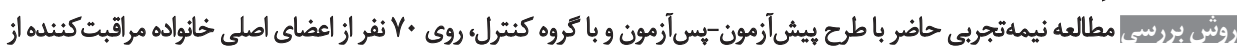

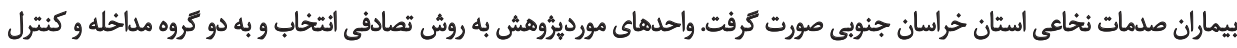

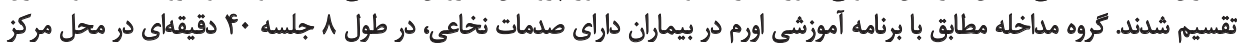

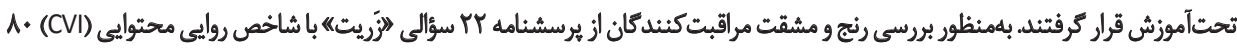

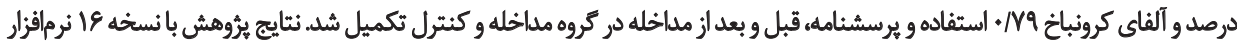

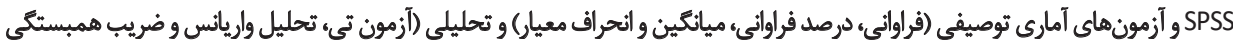
بيرسون) مورد تجزيه و تحليل قرار كرفتي.

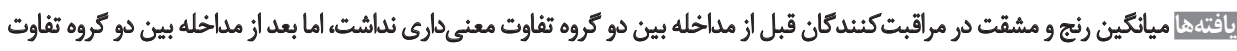

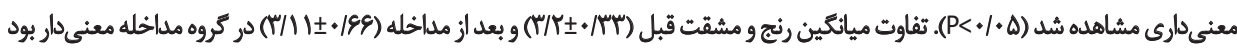

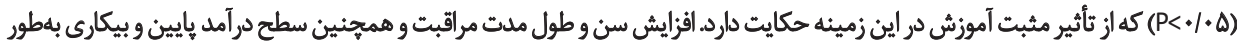

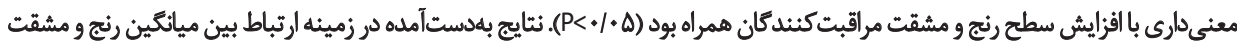

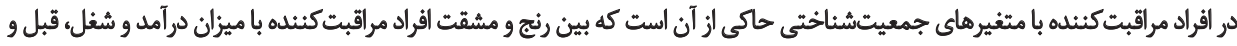

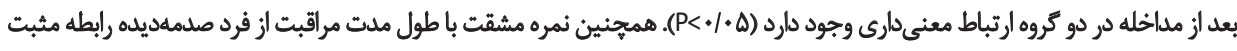

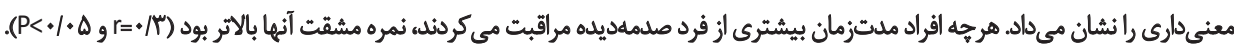

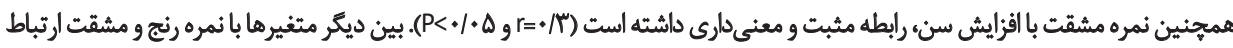

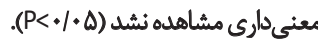

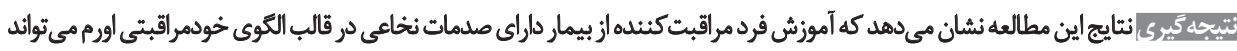

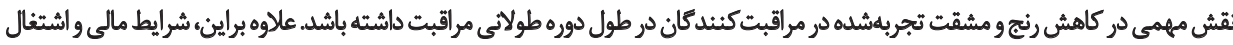

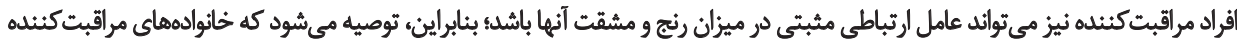

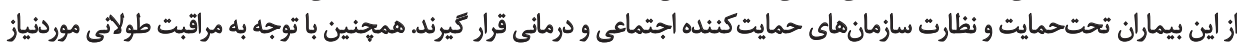

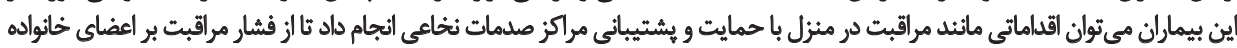
مراقبت كنيده كاستئه شود.

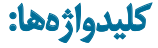

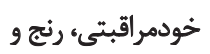
مشتقت، عضو خانواده مراقبت كنئده، صلدمات

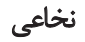


مراقبت كنيده زجرآور مىسازد. آنها فرصت كمى براي مراقبت از

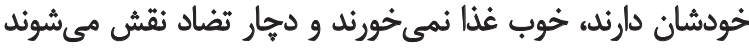

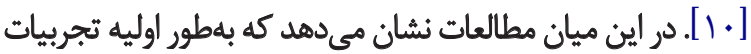

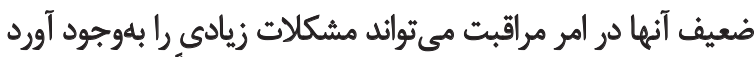

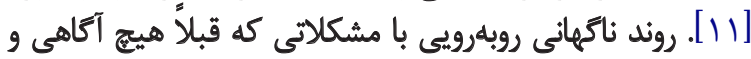

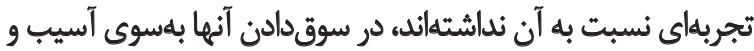

$$
\text { رنج نقش مؤثرى دارد. }
$$

عامل مشاركت كننده در رنج افراد مراقبت كنثنده، درك و ميزان

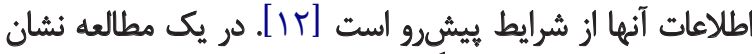

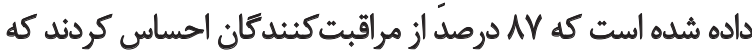

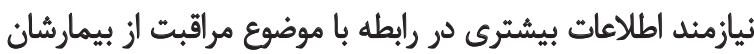

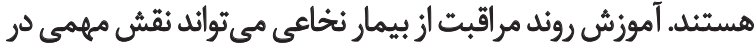

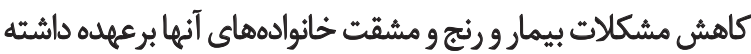

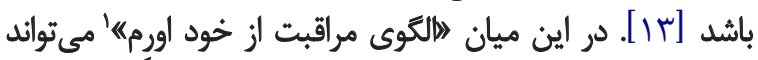

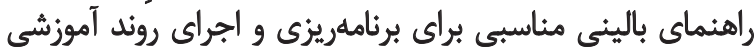

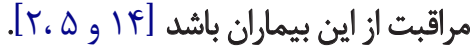

مرور متون مطالعات حاكى از اين است كه مراقبت كنيندكان

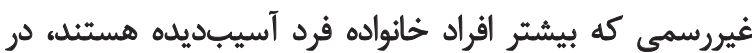

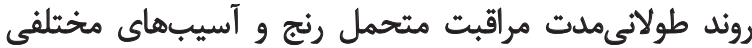

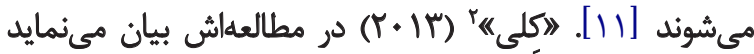

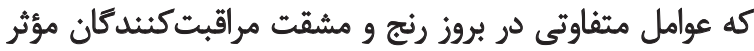

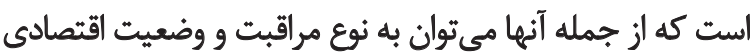

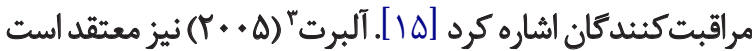

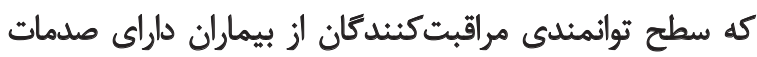

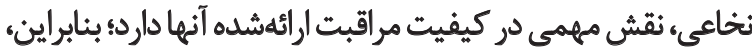

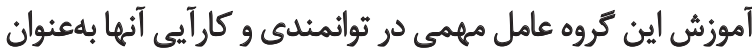

مراقبت كنئده بهحساب مى آيد [T]

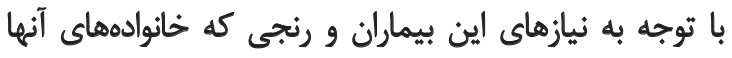

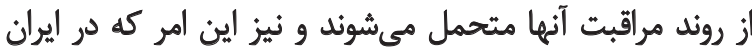

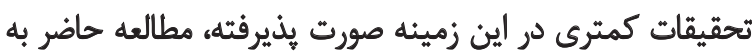

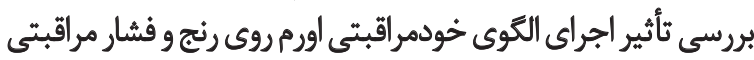
مراقبت كنئدكان اين بيمار ان برداخته است.

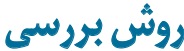

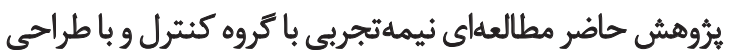

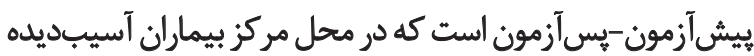

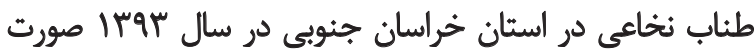

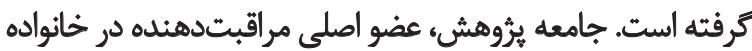

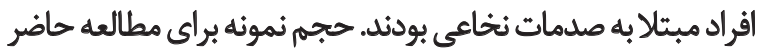

1. Orem self-care mode

2. Kelli

3. Albert
مשدمه

آسيبديدگى طناب نخاعى، حادثهاى فاجعلبار است كه مي تواند

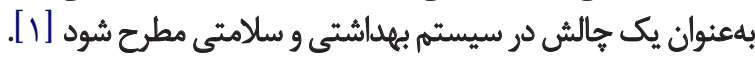

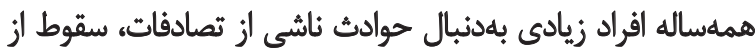

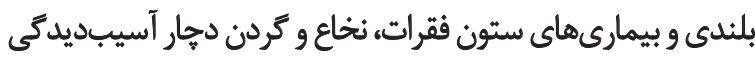

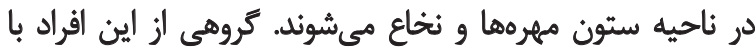

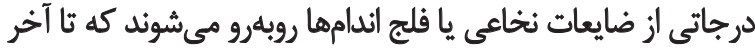

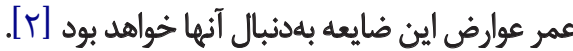

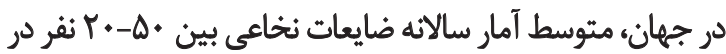

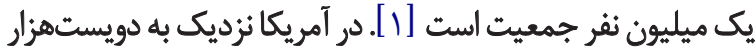

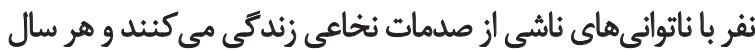

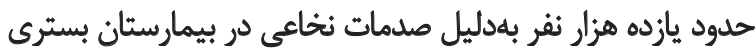

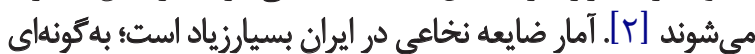

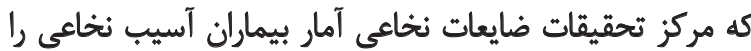

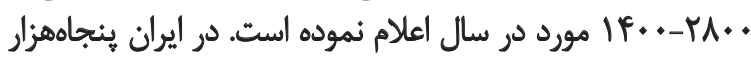

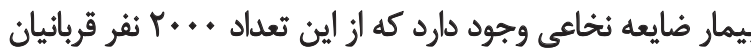
جنك تحميلى و بقيه در اثر عواملى نظير تصادف، سقوط از إز ارتفاع و... دجار آسيب نخاعى شدهاند [بوب].

آسيب طناب نخاعى باعث عوارض حسى و حركتى وسيعى

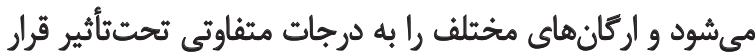

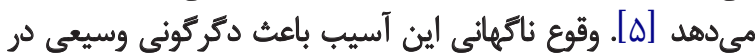

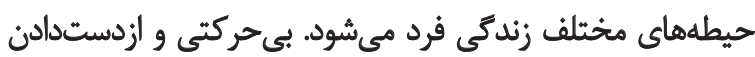

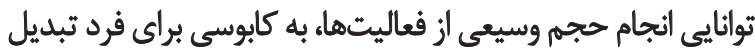

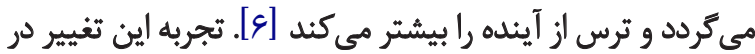

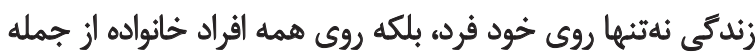

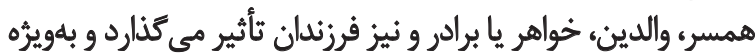

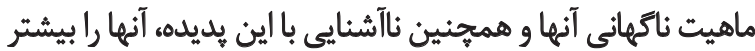

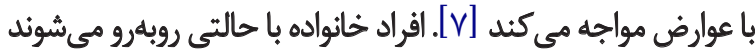

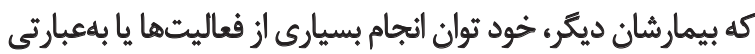

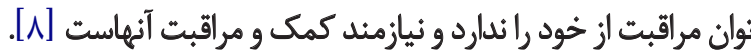
ناتوانى افراد براى مراقبت از خودشان مستلزم تلاشهاى افراد

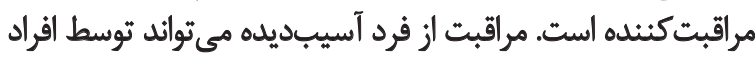

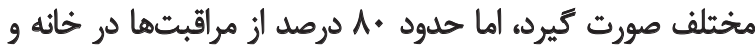

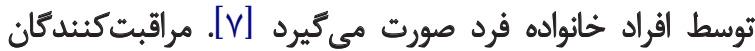

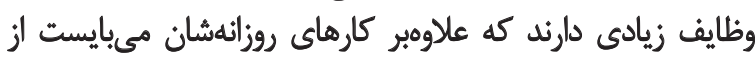

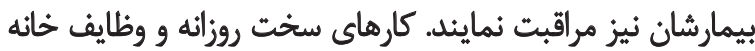

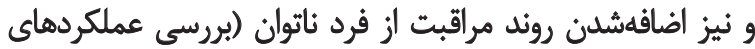

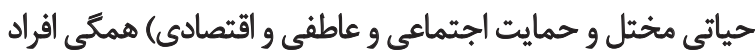

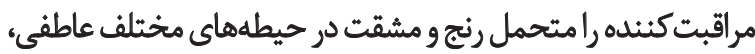

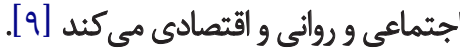

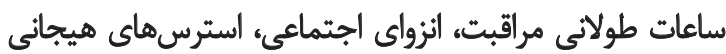

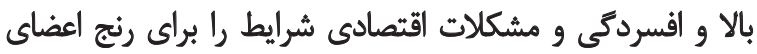


(هميشه) تعيين شده است [Tr]

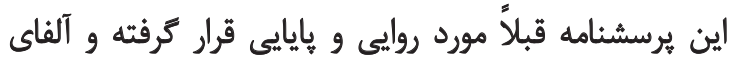

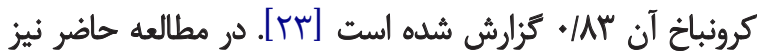

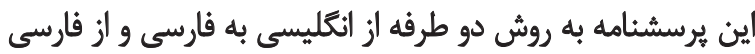

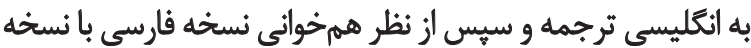

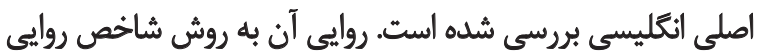

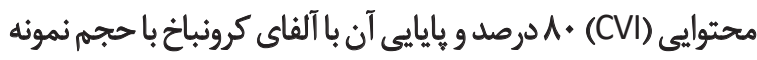

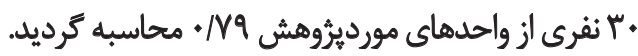

مداخله در اين يُروهش شامل آموزش به مراقبت كنيندكان در

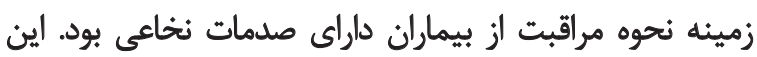

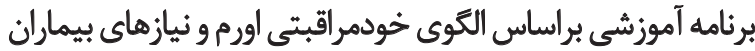

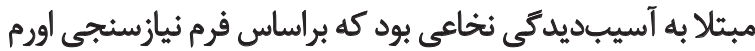

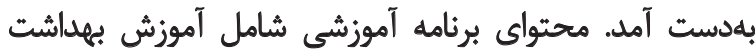

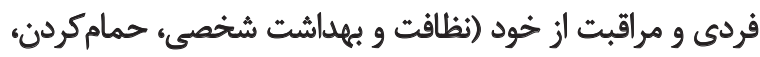

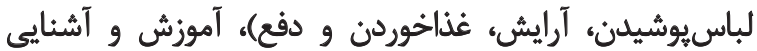

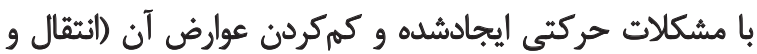

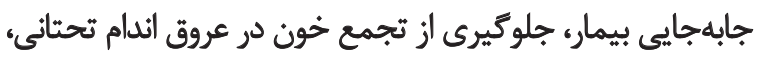

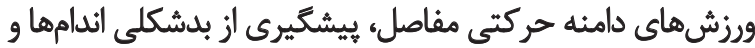

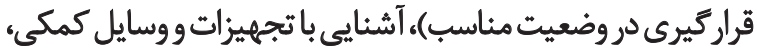

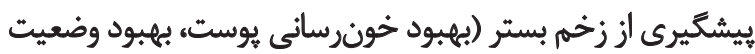

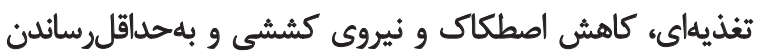

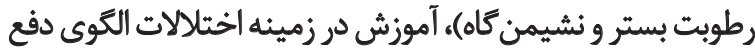

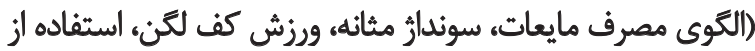

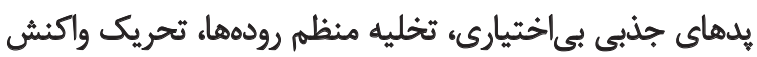

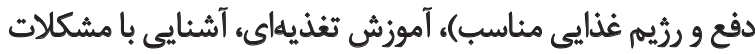

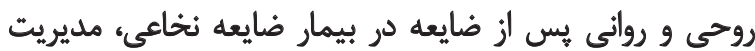

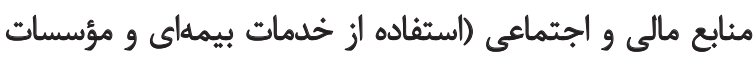

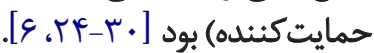

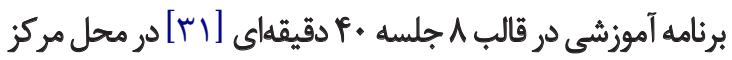

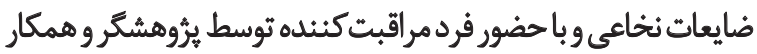

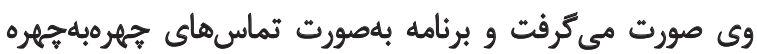

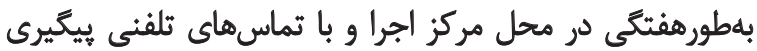

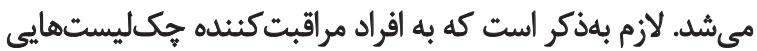

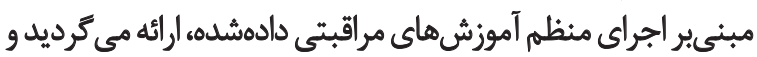

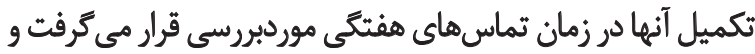

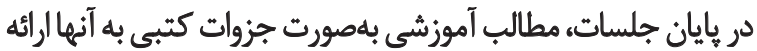

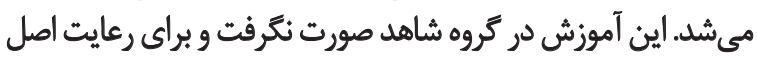

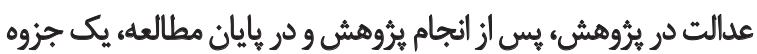

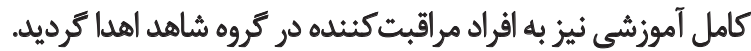

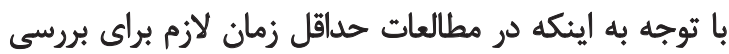

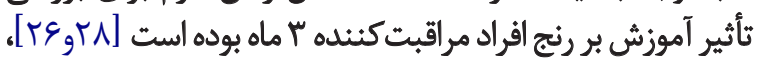

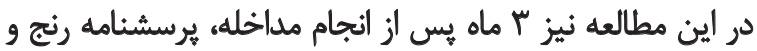

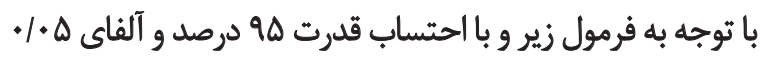

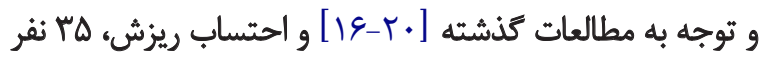
براى هر تروه در نظر ترفته شد.

$$
n=\left(s_{1}^{2}+s_{2}^{2} / x_{2}-x_{1}\right) f(\alpha+B)
$$

آمار كل بيماران نخاعى در مركز موردنظر • ل1 1 نفر بود. اين بيماران

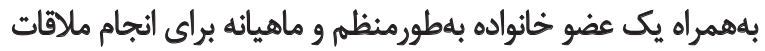

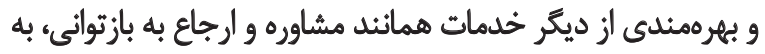

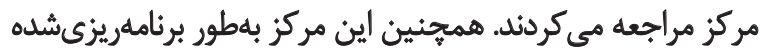

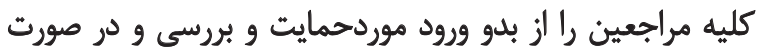

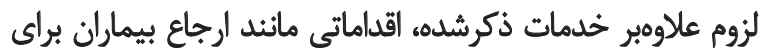

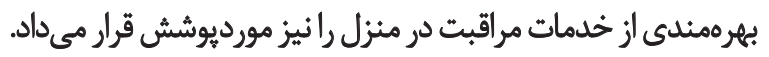
تعداد كل بيمارانى كه در انجمن ضايعات نخاعى عضو بودند

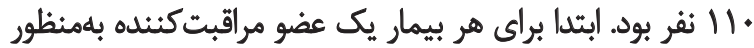

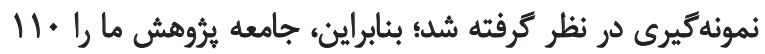

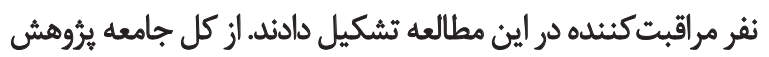

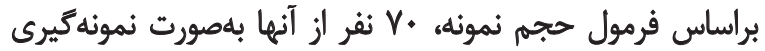

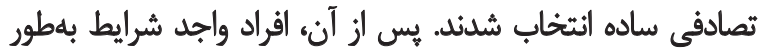

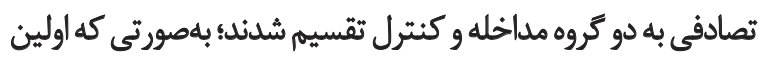

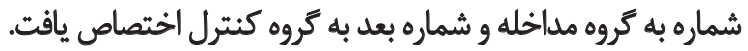
معيارهاي ورود به برؤوهش عبارت بود ازئ عضو درجه يك خانواديه،

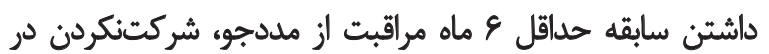

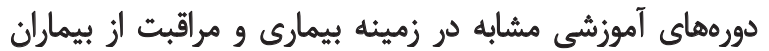

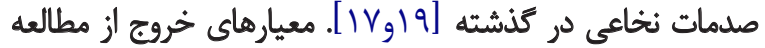

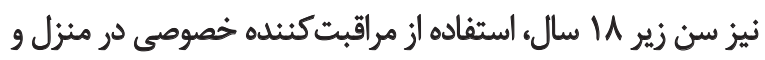
داشتن بيمارى روانى تشخيص داده شده بود.

يس از تخصيص تصادفى، عضو اصلى مراقبت كنئده از فرد بيمار

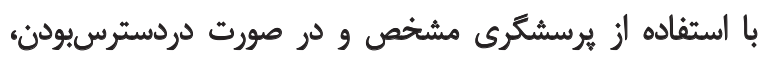

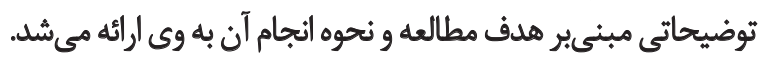

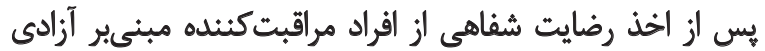

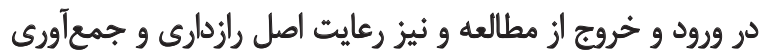

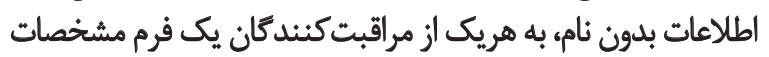

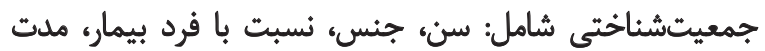

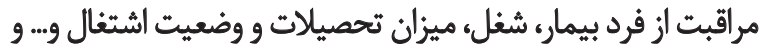
يرسشنامه رنج و مشقت در افراد مراقبت كنينده ارائه شد.

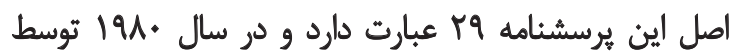

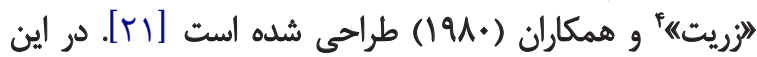

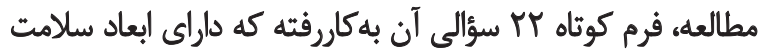

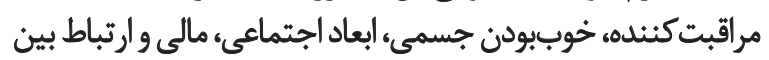

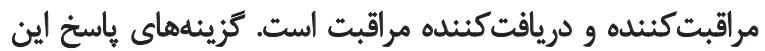

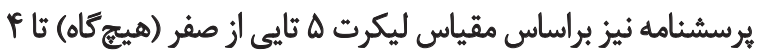




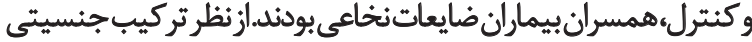

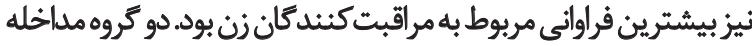

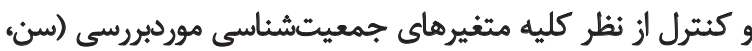

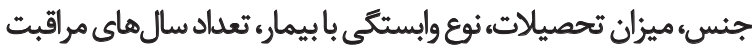

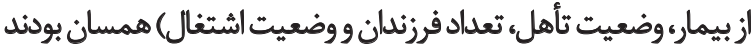

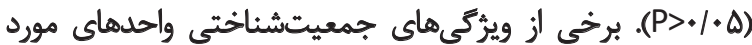
يروهش در جدول شماره ا بيان شده است. مياتَّين نمره رنج و مشقت افراد مراقبت كنينده قبل از مداخله در

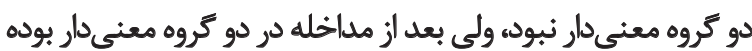

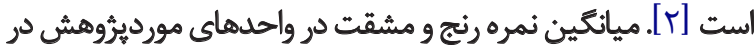
كروه مداخله قبل و بعد از مداخله تفاوت معند

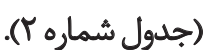

مشقت به افراد مراقبت كننده در هر دو كروه مداخله و كنترل

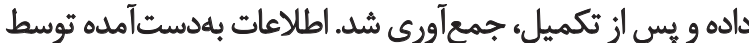

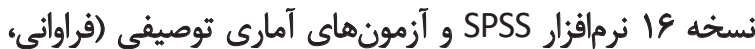

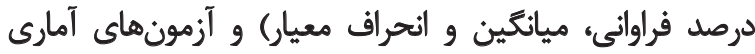

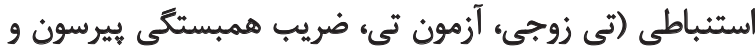
تحليل واريانس) مورد تجزيه و تحليل قرار ترفت

ياقتهنا

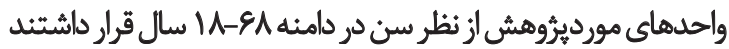

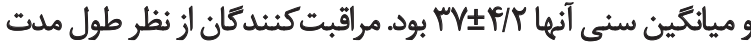

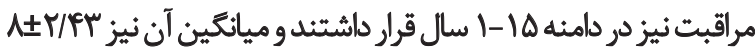
سال بود.ازنظر نوع وابستّى بيشتر افراد مراقبت كنينده در كروه مداخله

جدول ا. ويرُكى هاى جمعيتشئاختى واحدهاي مورديُوهش در دو كروه مداخله و كنترل.

\begin{tabular}{|c|c|c|c|c|c|c|}
\hline \multirow{2}{*}{ سطح معنى $ا$ رى } & \multicolumn{2}{|c|}{ كتثرل } & \multicolumn{2}{|c|}{ مداخليك } & & \multirow{2}{*}{ متغير } \\
\hline & نرصد & ت تعداد & درصد & تعداد & & \\
\hline \multirow{3}{*}{$\begin{array}{l}t / * r \\
t=m / m\end{array}$} & 8 & $M$ & VI/TY & ro & زن & \multirow{3}{*}{ جنس } \\
\hline & $r$. & if & rNOY & 1. & مرد & \\
\hline & 10. & ra & 1.0 & ra & كل & \\
\hline \multirow{5}{*}{$\begin{array}{c}.11 \\
F=1 F / 1\end{array}$} & IV/IF & 8 & $11 / 4 r$ & $f$ & ليىسواد & \multirow{5}{*}{ ميزان تحصيلات } \\
\hline & $1 F / r A$ & $\Delta$ & Tr/As & A & زيردييلم & \\
\hline & rV/IF & ir & $\mathrm{H} / \mathrm{TH}$ & 11 & دييلهم & \\
\hline & $T Y / A \Delta$ & $\wedge$ & TH/AS & A & ليسائس & \\
\hline & NoY & r & W/Tr & r & بالاتر & \\
\hline \multirow{3}{*}{$\begin{array}{c}.198 \\
F=F / / A\end{array}$} & IV/IF & 8 & TNAI & 1. & كمتر از ه....... & \multirow{3}{*}{ ميزان درآعد } \\
\hline & 8. & $M$ & $\Delta F / T A$ & 19 & $0 \ldots \ldots+1 \ldots \ldots$ & \\
\hline & Tr/AD & $\wedge$ & IV/IF & 8 & بالاتر از ........ 1.. & \\
\hline \multirow{2}{*}{. } & \multirow{2}{*}{ FNDY } & IV & & & & \multirow{3}{*}{ شعل فعلى } \\
\hline & & $M$ & $\pi / \pi$ & $\pi$ & بيغار & \\
\hline$t=r / h$ & $\Delta 1 / F T$ & $\Delta U / P T$ & $\operatorname{s\Delta M}$ & $m$ & 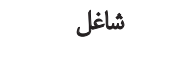 & \\
\hline.$/ 1 Y$ & $F r / T$ & 11 & r. & If & و والدين & \multirow{3}{*}{ نه به وابستكى بيمار } \\
\hline \multirow{2}{*}{$F=9 / 11$} & $\Delta / / 4 T$ & in & Pr/AQ & 10 & لهمبر & \\
\hline & IV/IF & 8 & IV/IF & 8 & فرزندان & \\
\hline .11 & $V M / F T$ & Ta & W/If & rV & بيملششله & \multirow{2}{*}{ وضعيت ييمه } \\
\hline$t=V / / f$ & $r \Delta / \Delta 1$ & 1. & Tr/As & A & فاقد بيمه & \\
\hline.$/ N$ & $P \Delta / Y$ & 18 & f. & If & شخصى & \multirow{2}{*}{ نوع مسكن } \\
\hline$t=r / q$ & $\Delta F / K A$ & 19 & \&. & $M$ & أستيجارى & \\
\hline$\cdot \pi$ & $r+$ & if & TNAI & 1. & بلى بل & استفاده از \\
\hline $\mathrm{t}=\Delta / \mathrm{t}^{\mathrm{t}}$ & 8. & $M$ & $V / N T$ & YA & خير & سيستمهاي حمايتى \\
\hline
\end{tabular}


جدول ب. مقايسه ميائكين رنج و مشقت واحدهاى مورديثوهش قبل و بعد از انجام مداخله بين دو كروه آزمون و كنترل.

\begin{tabular}{|c|c|c|c|c|}
\hline 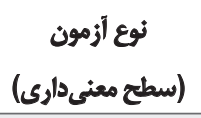 & 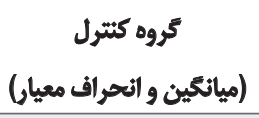 & 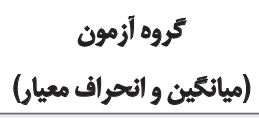 & مرحله & مثغير \\
\hline $\begin{array}{l}(P=+/ 1) \\
t=\varnothing / M A \\
(P=\odot / \bullet)^{*} \\
t=\Delta / \Delta\end{array}$ & $4 / 11 \pm . / 89$ & $\Pi / \pi \pm \cdot / \pi$ & قبل از مداخله & رثج و مشقت \\
\hline
\end{tabular}

جدول لا. مقايسه ميائكين نمره رنج و مشقت واحدهاي مورديروهش قبل و بعد از مداخله در هر كروه.

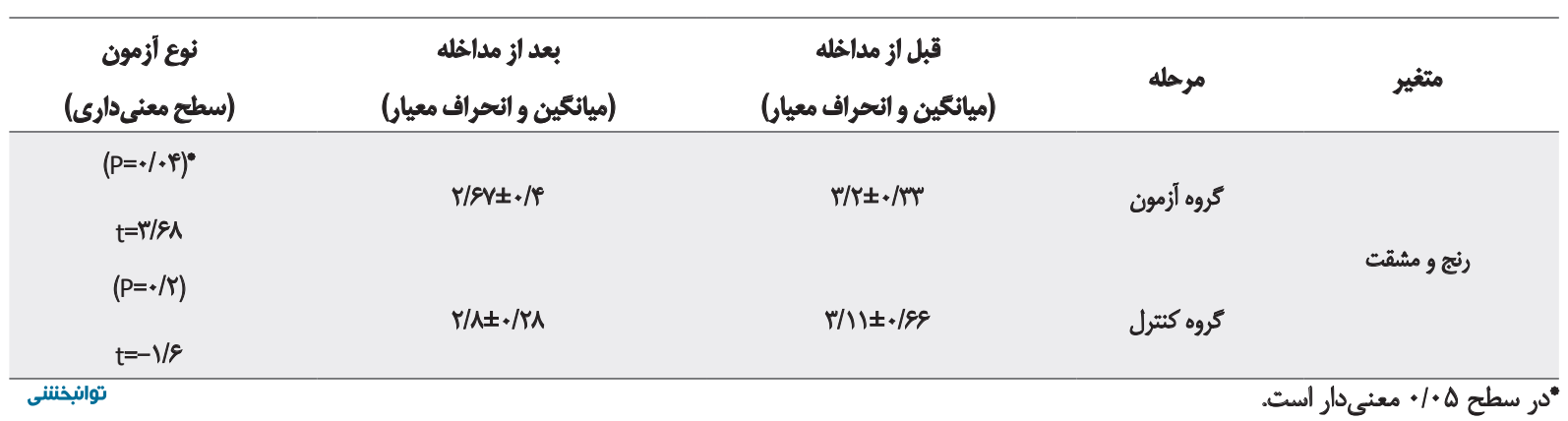

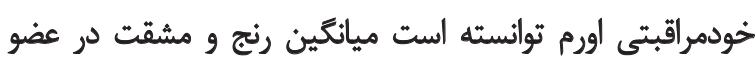

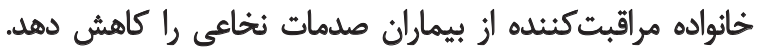

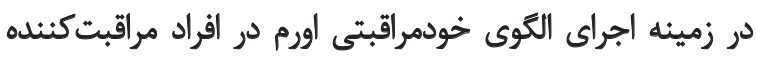
از بيماران داراى صدمات نخاعى مطالعهاى مشاهده نشده است أست

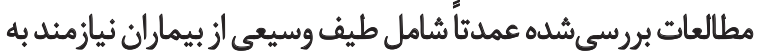
دريافت مراقبت خانكى بوده است.

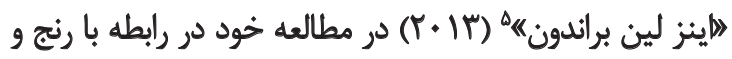

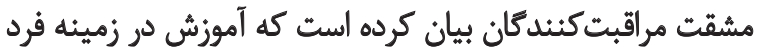

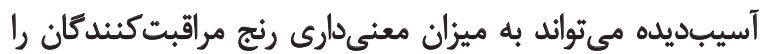

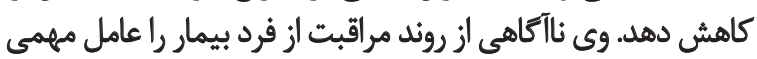

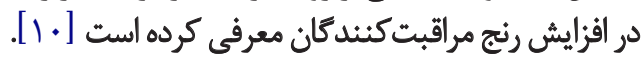

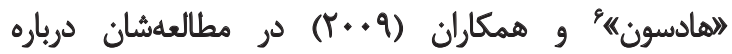

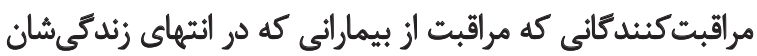

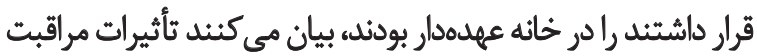

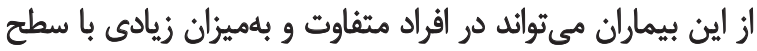

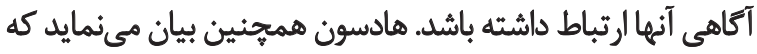

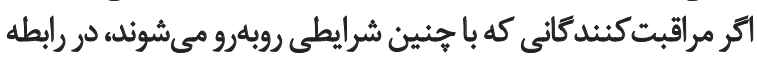

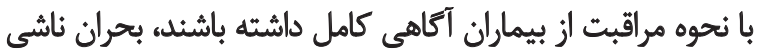

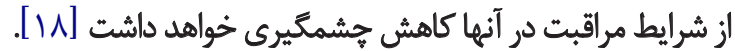

5. Inez Lynne Brandon 6. Hudson
نتايج بلهدتآمده در زمينه ارتباط بين ميانكين رنج و مشقت در

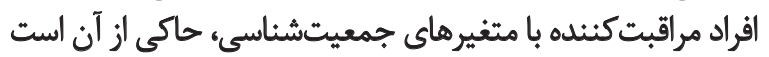

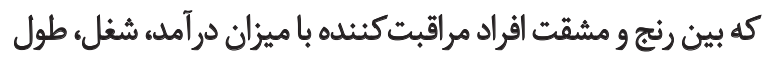

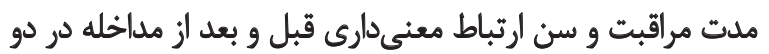

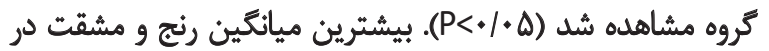

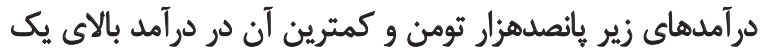

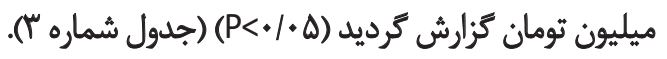
ارتباط نمره مشقت با شغل نيز نشان داد كه افراد بيكار يا ازكارافتاده

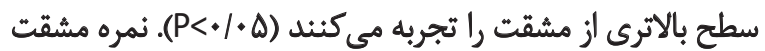

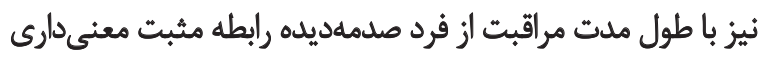

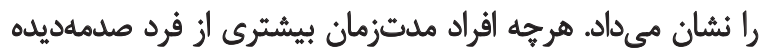

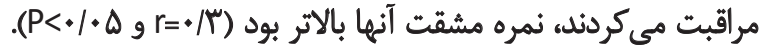

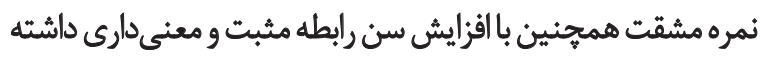

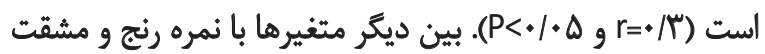

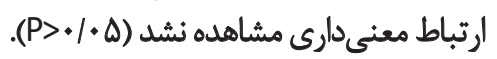

بين متغير مشقت با جنس (PAD)

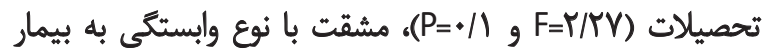

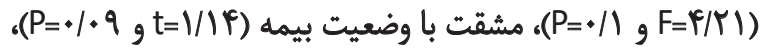

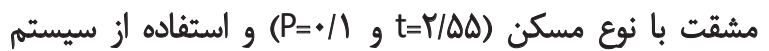
حمايتى (P= ) رابطه معنى $\stackrel{\leftrightarrow}{\oplus}$ نثايج اين مطالعه حاكى از آن است كه آموزش مطابق با الكوى 
أرائهده توسط مراقبت كننده را مدنظر قرار دادند و اذذعان داشتيند

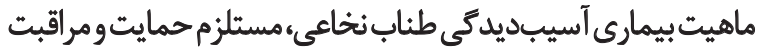

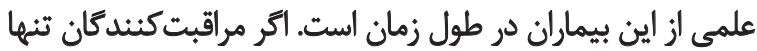

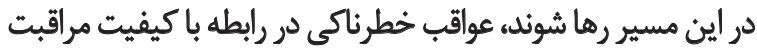

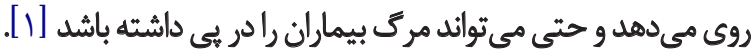
مطالعات صورت مرفته هريك به بررسى حيطه مثفاوتى از نقش

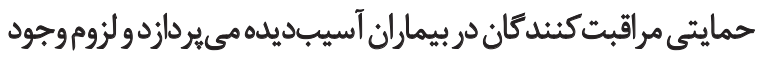

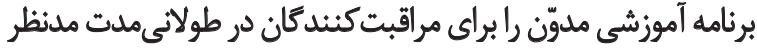

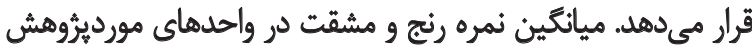
قبل از مطالعه در كروه مداخله و كروه آزمون بالا بوده است.

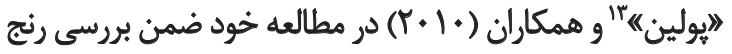
و مشقت و آسيبهاى روائى در همسران مراقبت كنينده از بيماران بران

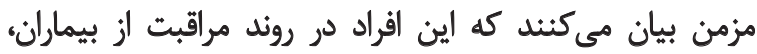

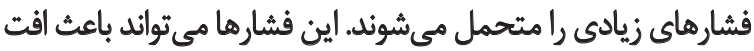

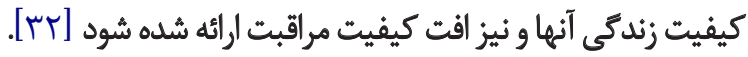

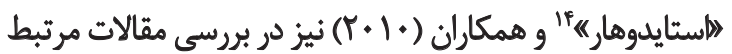

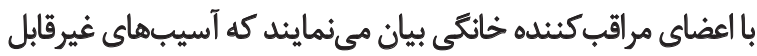

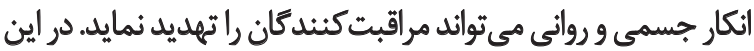

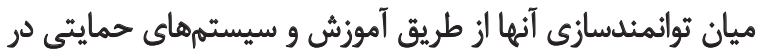

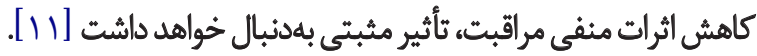

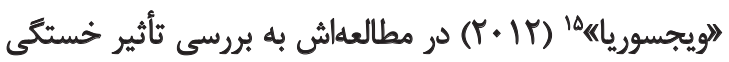

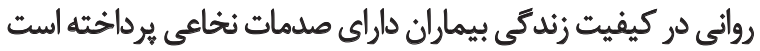

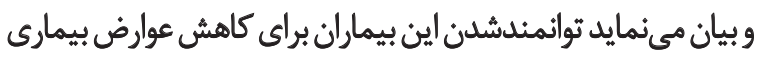

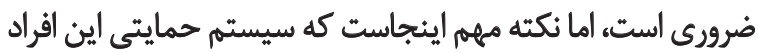

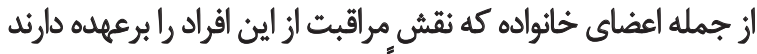

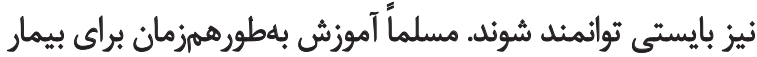

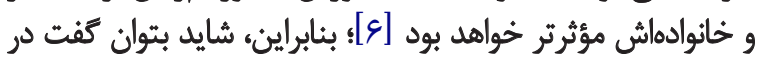

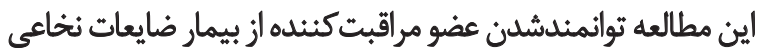

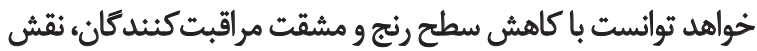

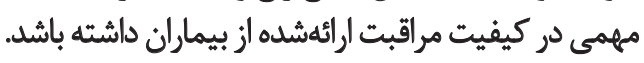

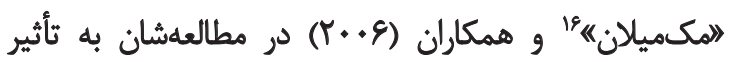

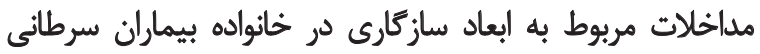

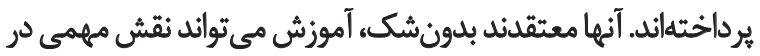

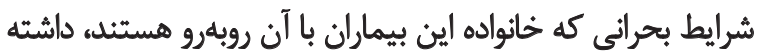

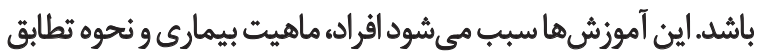

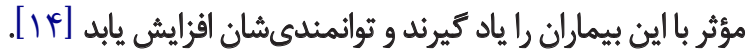
در زميئه ارتباط بين رنج و مشقت مراقبتكنيده با متغيرهاى

13. Poulin

14. Stajduhar

15. Wijesuriya

16. McMillan

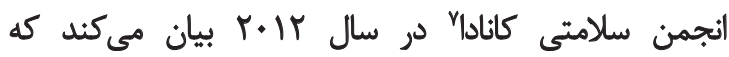

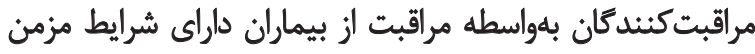

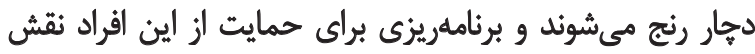

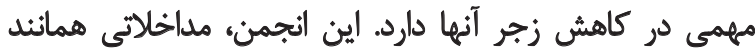

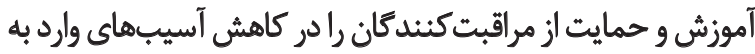

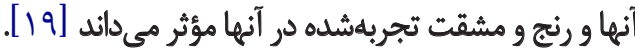

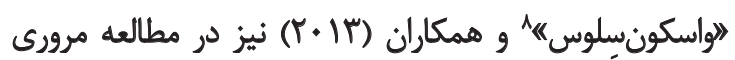

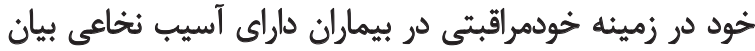

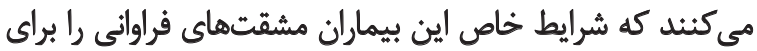

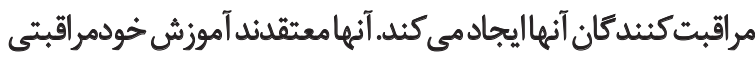

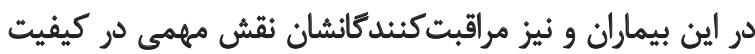

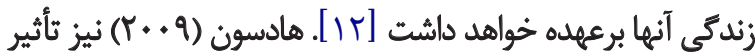

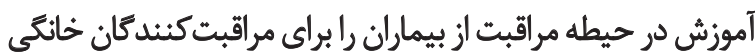

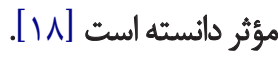

"امكلنون" (11) (r.11) در مطالعهاش در مورد رنج همسران

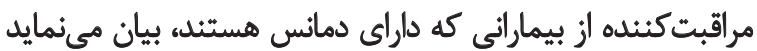

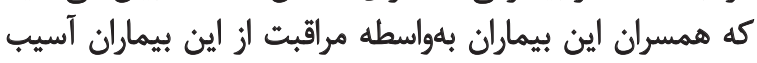

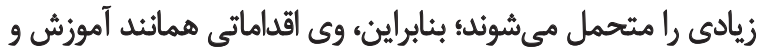

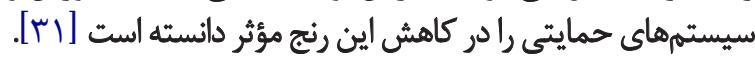

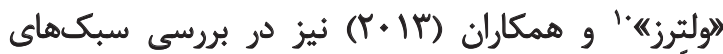

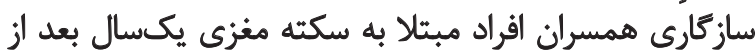

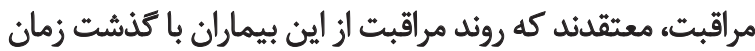

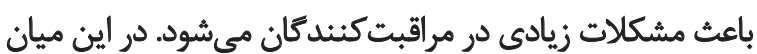

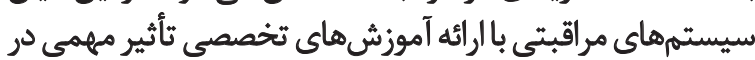

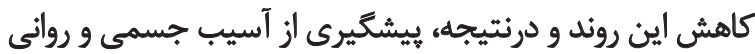

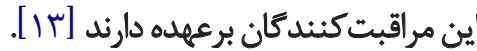

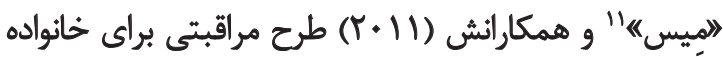

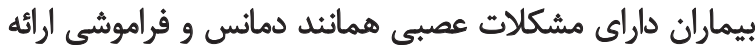

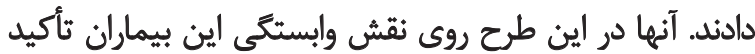

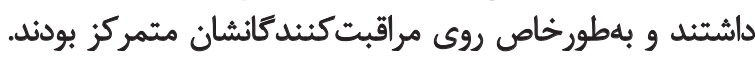

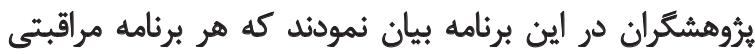

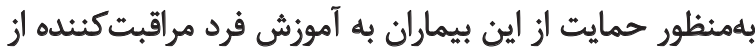

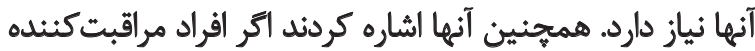

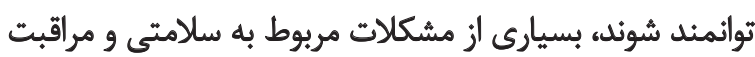

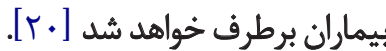

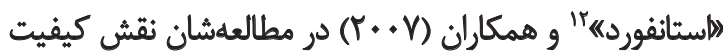

7. Health Council of Canada

8. Vasconcelos

9. McLennon

10. Wolters

11. Mace

12. Stanford 


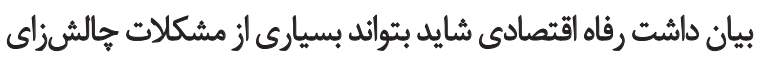

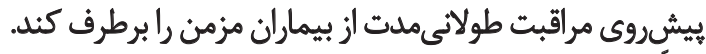

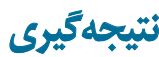

نتايج مطالعه حاضر حاكى از آن است كه آموزش افراد آنداد

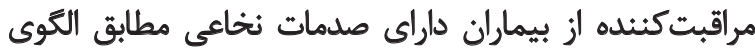

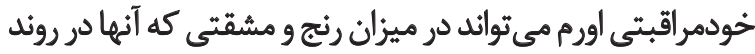

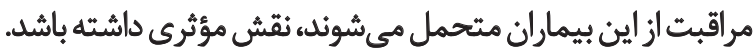

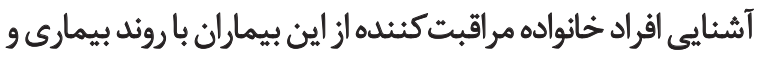

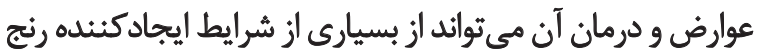

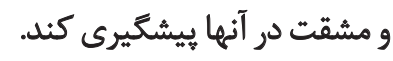

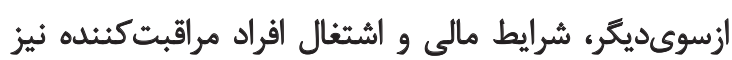

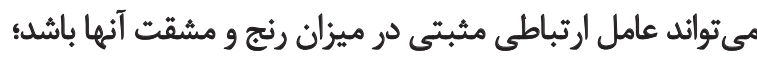

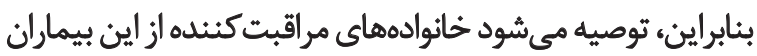

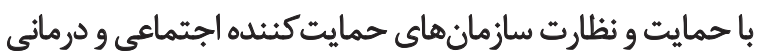

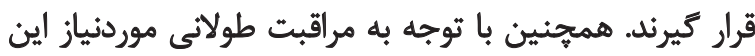

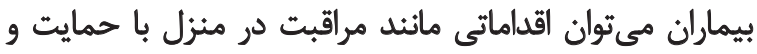

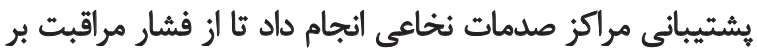
العضاى خانواده مراقبت كنينده كاسته شود.

\section{از محدوديتهاى اين مطالعه مى توان به اين نكته اشاره كرد كه}

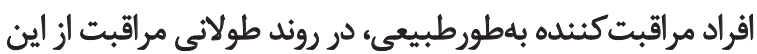

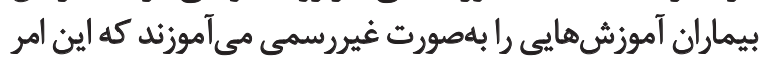

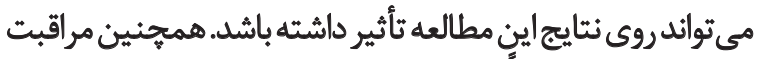

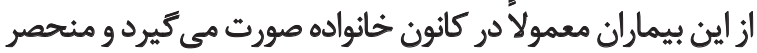

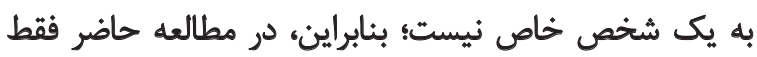

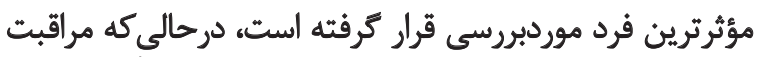

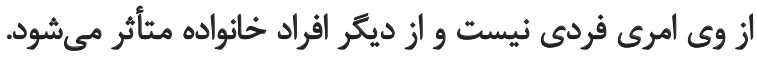

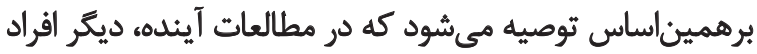

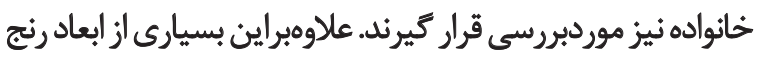

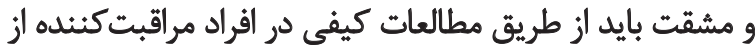

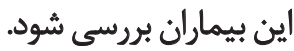

$$
\text { تشكر و قدر داني تئي }
$$

در خايان از كليه همكاران و نيز افراد خانوادههاى داراى بيماران صدمات نخاعى كه صميمانه در اين مطالعه همكارى دانئ داشتيند،

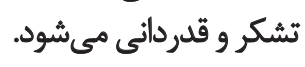

جمعيتشناسى، نتايج حاكى از آن است كه بين سن مراقبت مثنده

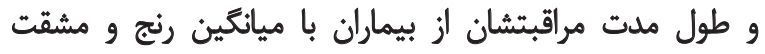

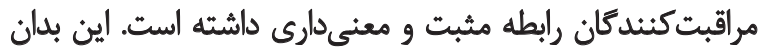

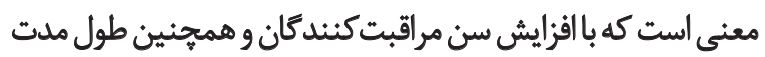

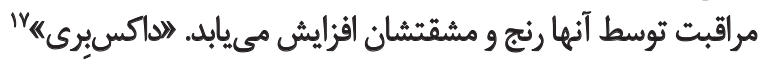

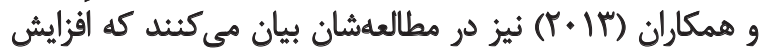

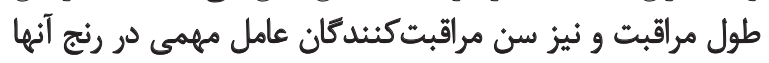

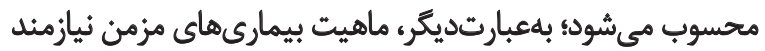

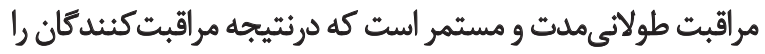

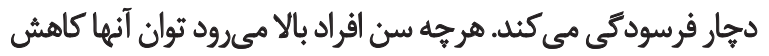

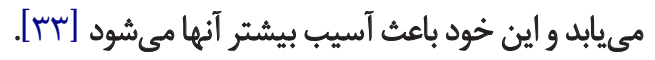

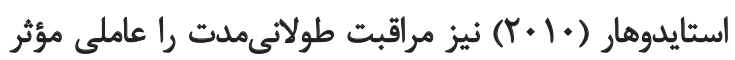

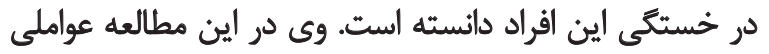

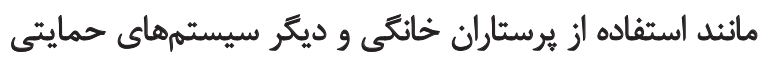

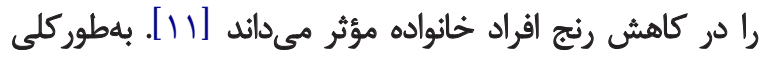

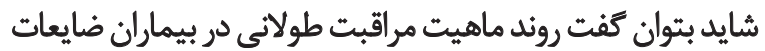

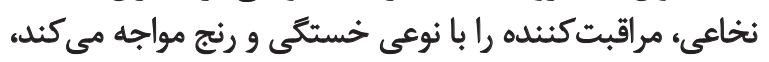

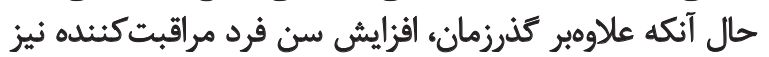

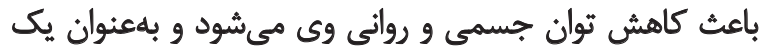

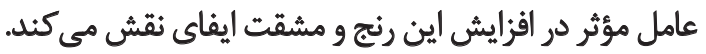
ميانكين نمره مشقت ورنج مراقبت كنيندكان با متغير شغل و ميزان

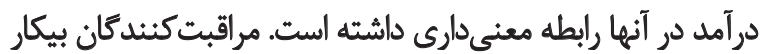

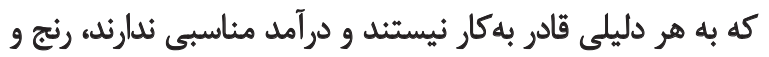

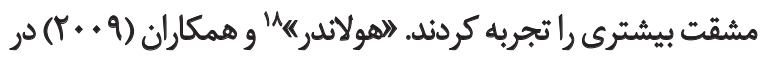

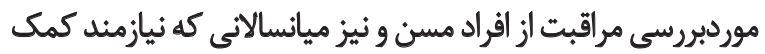

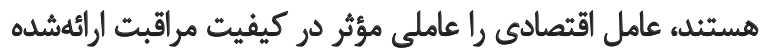

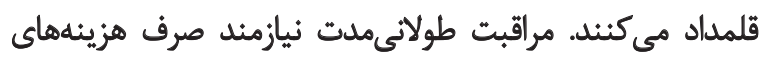

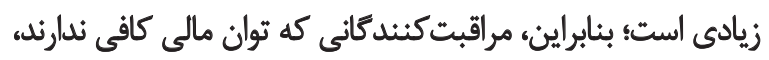
متحمل آسيب خواهند شد [MF]

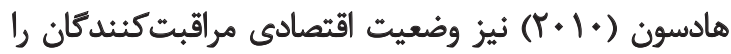

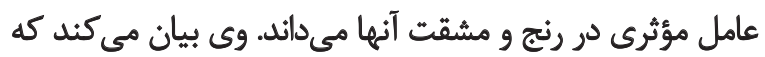

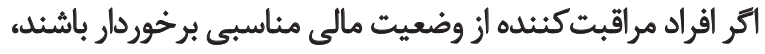

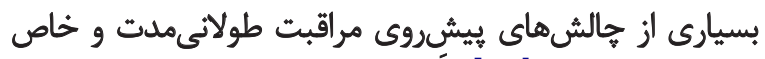

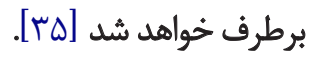

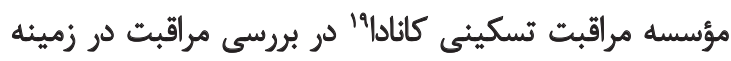

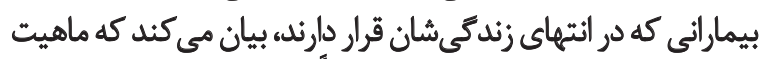

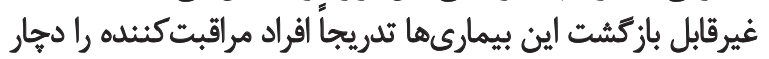

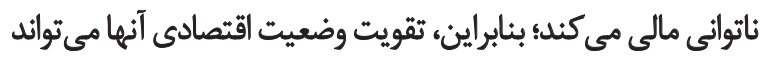

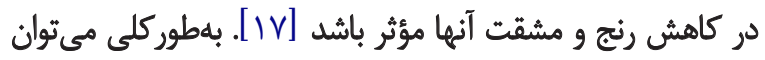

17. Duxbury

18. Hollander

19. Canadian Hospice and Palliative Care Association 


\section{References}

[1] Stanford RE, Soden R, Bartrop R, Mikk M, Taylor TKF. Spinal cord and related injuries after attempted suicide: Psychiatric diagnosis and longterm follow-up. Spinal Cord. 2007; 45(6):437-43.

[2] Albert T, Ravaud JF. Rehabilitation of spinal cord injury in France: A nationwide multicentre study of incidence and regional disparities. Spinal Cord. 2005; 43(6):357-65.

[3] Noreau L, Fougeyrollas P. Long-term consequences of spinal cord injury on social participation: The occurrence of handicap situations. Disability and Rehabilitation. 2000; 22(4):170-80.

[4] Kashee A. [The guideline of people with spinal cord injury (Persian)]. $1^{\text {st }}$ ed. Tehran: Research Center of Veterans Engineering and Medical Sciences; 2009.

[5] Bauchet L, Lonjon N, Perrin FE, Gilbert C, Privat A, Fattal C. Strategies for spinal cord repair after injury: A review of the literature and information. Annals of Physical and Rehabilitation Medicine. 2009; 52(4):330-51.

[6] Wijesuriya N, Tran Y, Middleton J, Craig A. The impact of fatigue on the health related quality of life in persons with spinal cord injury. Archives of Physical Medicine and Rehabilitation. 2012; 93(2):319-324.

[7] National Alliance for Caregiving. Care for the family caregiver: A place to start [Internet]. New York: Emblem Health; 2010. Available from: http://www.caregiving.org/data/Emblem_CfC10_Final2.pdf.

[8] Schulz R, Beach SR. Caregiving as a risk factor for mortality: The caregiver health effects study. Journal of the American Medical Association. 1999; 282(23):2215-19.

[9] Bloemen Vrencken JH, de Witte LP, Post MW, van den Heuvel WJ. Health behaviour of persons with spinal cord injury. Spinal Cord. 2007; 45(3):243-9

[10] Brandon IL. Easing the burden on family caregivers. Nursing. 2013; 43(8):36-42

[11] Stajduhar KI, Funk L, Toye C. Home-based family caregiving at the end of life: A comprehensive review of published quantitative research (1998-2008). Palliative Medicine. 2010; 26(6):573-593.

[12] Vasconcelos AS, França ISX, Coura AS, Enders BC, Cartaxo HGO, Sousa FS. Self-care in neurogenic intestine in subjects with spinal cord injury: An integrative review. Online Brazilian Journal of Nursing [Internet]. 2013 [cited 2013 Dec 22]; 12(4):998-1010. doi: 10.5935/1676-4285.20133692. Available from: http://www. objnursing.uff.br/index.php/nursing/article/view/3692.

[13] Wolters Gregorio G, Visser Meily JM, Tan FE, Post MW, van Heugten CM. Changes in the coping styles of spouses and the influence of these changes on their psychosocial functioning the first year after a patient's stroke. Journal of Psychosomatic Research. 2011; 71(3):188-193.

[14] McMillan SC, Small BJ, Weitzner M, Schonwetter R, Tittle M, Moody L, et al. Impact of coping skills intervention with family caregivers of hospice patients with cancer: A randomized clinical trial. Cancer. 2006; 106(1):214-22.
[15] Kelli IS. Burdens of Family Caregiving at the End of Life. Clinical and Investigative Medicine. 2013; 36(3):121-126.

[16] Pocock SJ. Clinical trial: A practical approach. New York: John Wiley \& Sons Publishing; 1983.

[17] Canadian Hospice Palliative Care association. The role of informal caregivers in hospice palliative and end-of-life care in Canada: A discussion of the legal, ethical and moral challenges [Internet]. Ottawa: CHPCA; 2004 [cited 2013 April 5]. Available from: http://www.chpca.net/media/7901/voice_project-discussion_document-August2004-2.pdf.

[18] Hudson P, Thomas K, Quinn K, Cockayne M, Braithwaite M. Teaching family carers about home based palliative care: Final results from a group education program. Journal of Pain and Symptom Management. 2009; 38(2):299-308.

[19] Health Council of Canada. Seniors in need, caregivers in distress [Internet]. Toronto, Ontario, Canada: Health Council of Canada; 2012 [cited 2013 May 13]. Available from: http://legion.ca/_pdf/ SBureau/Seniors\%20in\%20Need\%20H CC_HomeCare_e.pdf.

[20] Mace NL, Rabins PV. The 36-hour day: A family guide to caring for people who have Alzheimer's disease, related dementias and memory loss. $5^{\text {th }}$ ed. Baltimore, MD: Johns Hopkins University Press; 2011.

[21] Zarit SH, Reever KE, Bach-Peterson J. Relatives of the impaired elderly: Correlates of feelings of burden. Gerontologist. 1980; 20(6):649-655

[22] Bédard M, Molloy W, Squire L, Dubois S, Lever JA, O'Donnell M. The Zarit Burden Interview: A New Short Version and Screening Version. Gerontologist. 2001; 41(5):652-657.

[23] Lai DWL. Validation of the Zarit Burden Interview for Chinese Canadian caregivers. Social Work Research. 2007; 31(1):45-53.

[24] International Association of Neurorestoratology (IANR). Spinal Cord Injury Functional Rating Scale, SCI-FRS (Seeking Suggestions). Beijing, China: International Association of Neurorestoratology (IANR); 2010.

[25] Hoenig H, Hoff J, McIntyre L, Branch LG. The Self-Reported Functional Measure: Predictive validity for health care utilization in multiple sclerosis and spinal cord injury. Archives of Physical Medicine and Rehabilitation. 2001; 82(5):613-8.

[26] Strauss DJ, DeVivo MJ, Paculdo DR, Shavelle RM. Trends in life expectancy after spinal cord injury. Archives of Physical Medicine and Rehabilitation. 2006; 87(8):1079-85.

[27] Anton HA, Miller WC, Townson AF. Measuring fatigue in persons with spinal cord injury. Archives of Physical Medicine and Rehabilitation. 2008; 89(3):538-42.

[28] Gelis A, Stefan A, Colin D, Albert T, Gault D. Therapeutic education in persons with spinal cord injury: A review of the literature. Annals of Physical and Rehabilitation Medicine. 2011; 54(3):189-210

[29] Wuermser LA, Ho CH, Chiodo AE, Priebe MM, Kirshblum SC, Scelza WM. Spinal cord injury medicine. Acute care management of traumatic and nontraumatic injury. Archives of Physical Medicine and Rehabilitation. 2007; 88(8):1083-5. 
[30] Silva NA, Sousa N, Reis RL, Salgado AJ. From basics to clinical: A comprehensive review on spinal cord injury. Progress in Neurobiology. 2014; 114:25-57.

[31] McLennon SM, Havermann B, Rice M. Finding meaning as a mediator of burden on the health of caregivers of spouses with dementia. Aging \& Mental Health. 2011; 15(4):522-530.

[32] Poulin MJ, Brown SL, Ubel PA, Smith DM, Jankovic A, Langa KM. Does a helping hand mean a heavy heart? Helping behaviors and well-being among spouse caregivers. Psychology and Aging. 2010; 25(1):108-117.

[33] Duxbury L, Higgins C, Schroeder B. Balancing paid work and caregiving responsibility: A closer look at family caregivers in Canada [Internet]. Canada: Canadian Policy Research Networks. 2009 [cited 2013 April 10]. Available from: http://www.cprn.org/ doc.cfm?doc $=1997 \& \mathrm{l}=\mathrm{en}$.

[34] Hollander MJ, Liu G, Chappell NL. Who cares and how much? The imputed economic contribution to the Canadian healthcare system of middle aged and older unpaid caregivers providing care to the elderly. Healthcare Quarterly. 2009; 12(2):42-59.

[35] Hudson PL, Remedios C, Omas K. A systematic review of psychosocial interventions for family carers of palliative care patients. BMC Palliative Care. 2010; 9:17. 